\title{
Solutions for Series of Exponential Equations in Terms of Lambert-W Function and Fundamental Constants
}

\section{S. Gnanarajan}

Aruja \& Arjun Pty Ltd., Sydney, Australia

Email: rajan.sgnanarajan@gmail.com

How to cite this paper: Gnanarajan, S. (2018) Solutions for Series of Exponential Equations in Terms of Lambert-W Function and Fundamental Constants. Journal of Applied Mathematics and Physics, 6, 725-736.

https://doi.org/10.4236/jamp.2018.64065

Received: March 1, 2018

Accepted: April 17, 2018

Published: April 20, 2018

Copyright $\odot 2018$ by author and Scientific Research Publishing Inc. This work is licensed under the Creative Commons Attribution International License (CC BY 4.0). http://creativecommons.org/licenses/by/4.0/

\section{Abstract}

Series of exponential equations in the form of $x=y^{n} y^{\left(\frac{x}{y^{n+1}}\right)}$ were solved graphically, numerically and analytically. The analytical solution was derived in terms of Lambert-W function. A general numerical solution for any $y$ is found in terms of $n$ or in base $y$. A solution $\frac{10^{2} W\left(\frac{-\ln 10}{10}\right)}{\left(-\frac{\ln 10}{10}\right)}=137.129$ is close to the fine structure constant. The equation which provided the solution as the fine structure constant was derived in terms of the fundamental constants.

\section{Keywords}

Exponential Equation, Lambert-W Function, Fine Structure Constant, Logarithmic Equation, Numerical Analysis, Fundamental Constants

\section{Introduction}

Exponential equations are widely used in natural and social sciences. In this paper, we considered series of exponential equations and solved them graphically, numerically, and analytically in terms of Lambert-W function. One equation connected to the fine structure constant, was derived in terms of the fundamental constants and led to a new equation. The Lambert-W function for real variables is defined by the equation $W(x) \exp [W(x)]=x$ [1] [2] [3] [4] and it has applications in Planks spectral distribution law [5] [6], QCD renormalization [7], solar cells [8], bio-chemical kinetics [9], optics [10], population growth 
and water movement in soil [11].

Considering the series of exponential equations defined by the following equation

$$
x=y^{n} y^{\left(\frac{x}{y^{n+1}}\right)}
$$

where $x, y, n$ are real variables.

Taking $\log _{y}$ on both sides of the Equation (1.1)

$$
\log _{y} x=n+\frac{x}{y^{n+1}}
$$

Converting the Equation (1.2) to natural logarithm

$$
\frac{\ln x}{\ln y}=\frac{x}{y^{n+1}}+n
$$

The trivial solution of the Equations (1.1) to (1.3) is

$$
x=y^{n+1}
$$

In this paper, we are focusing on the non-trivial solutions.

For $n=2,1,0,-1,-2$, the Equations (1.1) and (1.3) become:

$$
\begin{gathered}
x=y^{2} y^{\frac{x}{y^{3}}} \text { or } \frac{\ln x}{\ln y}=\frac{x}{y^{3}}+2 \\
x=y y^{\frac{x}{y^{2}}} \text { or } \frac{\ln x}{\ln y}=\frac{x}{y^{2}}+1 \\
x=y^{\frac{x}{y}} \text { or } \frac{\ln x}{\ln y}=\frac{x}{y} \text { or } x^{y}=y^{x} \\
x=y^{-1} y^{x} \text { or } \frac{\ln x}{\ln y}=x-1 \\
x=y^{-2} y^{x y} \text { or } \frac{\ln x}{\ln y}=x y-2
\end{gathered}
$$

\section{Graphical Solutions}

If $y=10$, the Equations (1.5) to (1.9) become

$$
\begin{gathered}
x=10^{2} \times 10^{\frac{x}{10^{3}}} \\
x=10 \times 10^{\frac{x}{10^{2}}} \\
x=10^{\frac{x}{10}} \\
x=10^{-1} \times 10^{x} \\
x=10^{-2} \times 10^{10 x}
\end{gathered}
$$

The curves and the straight line to obtain the graphical solutions of the Equations (2.1) to (2.5) are shown in Figure 1. The intercepts of the curves and the straight line indicate the solutions. 


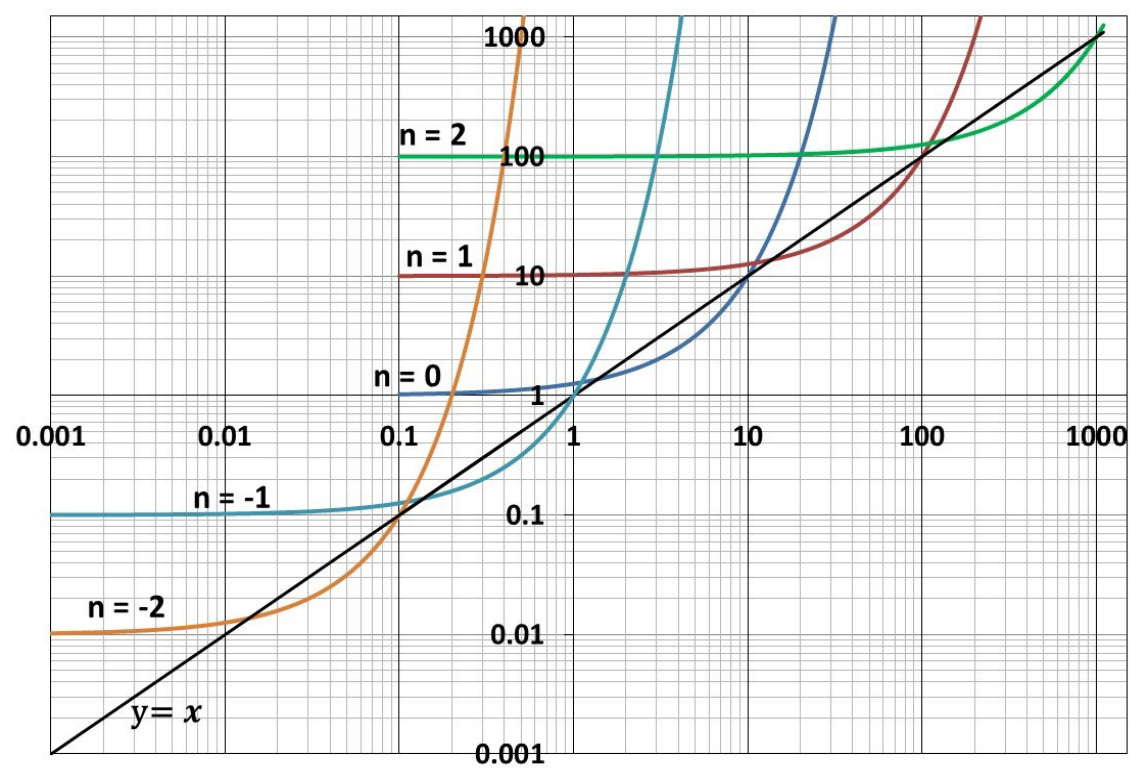

Figure 1. Plots of the functions to obtain the graphical solutions for the Equations (2.1) to $(2.5)$.

The intersecting points of $0.1,1,10,100$ and 1000 are the trivial solutions and the intersecting points at around $0.0137,0.137,1.37,13.7$ and 137 are the non-trivial solutions.

The non-trivial solutions imply the following equations:

$$
\begin{gathered}
10^{0.1371}=1.371 \\
\frac{\ln 1.371}{1.371}=\frac{\ln 10}{10}=0.2302 \\
10^{1.371}=1.371^{10}=23.5
\end{gathered}
$$

\section{Numerical Solutions}

Higher precision non-trivial numerical solutions were obtained for the series of equations $x=y^{n} y^{\left(\frac{x}{y^{n+1}}\right)}$ using the iterative technique for $n=2,1.5,1,0.5,0$, $-0.5,-1,-2$ and $1 \leq y \leq 15$ (Table 1 ). The iterations do not converge on non-trivial solutions for $y<e$, and solutions in this range were obtained by trial and error.

The solutions in Table 1 for $n=-2,-1,0,0.5,1,2$ are plotted as $x$ vs $y$ with $x$ axis in log scale (Figure 2). Sharp turning points in the plots are observed for $y$ values in the range of 1 to 2 .

\section{Analytical Solution}

Consider the Equation (1.3)

$$
\frac{\ln x}{\ln y}=\frac{x}{y^{n+1}}+n
$$


Table 1. Non-trivial numerical solutions for the series of equations $x=y^{n} y^{\left(\frac{x}{y^{n+1}}\right)}$.

\begin{tabular}{|c|c|c|c|c|c|c|c|c|}
\hline \multirow{2}{*}{$y$} & \multicolumn{8}{|c|}{ Solutions $x$ for different $n$ values } \\
\hline & -2 & -1 & -0.5 & 0 & 0.5 & 1 & 1.5 & 2 \\
\hline 1.1 & 36 & 39.6 & 41.53 & 43.56 & 45.68 & 47.92 & 50.25 & 52.71 \\
\hline 1.3 & 7.41 & 9.63 & 10.99 & 12.53 & 14.28 & 16.28 & 18.56 & 21.17 \\
\hline 1.5 & 3.29 & 4.94 & 6.05 & 7.41 & 9.07 & 11.11 & 13.61 & 16.67 \\
\hline 2 & 1 & 2 & 2.83 & 4 & 5.66 & 8 & 11.31 & 16 \\
\hline$e$ & 0.366937 & 1.00000 & 1.644494 & 2.718282 & 4.46877 & 7.3890 & 12.13412 & 20.0855 \\
\hline 3 & 0.275339 & 0.82601 & 1.430704 & 2.478052 & 4.292113 & 7.4341 & 12.87634 & 22.3024 \\
\hline 4 & 0.125 & 0.500000 & 1 & 2.000000 & 4 & 8.0000 & 16 & 32.0000 \\
\hline 5 & 0.070597 & 0.352984 & 0.789297 & 1.764922 & 3.946485 & 8.8246 & 19.73243 & 44.1230 \\
\hline 6 & 0.045118 & 0.270707 & 0.663095 & 1.624244 & 3.978569 & 9.7454 & 23.87141 & 58.4727 \\
\hline 7 & 0.031227 & 0.218591 & 0.578339 & 1.530140 & 4.04837 & 10.7109 & 28.33859 & 74.9768 \\
\hline 8 & 0.022852 & 0.182813 & 0.517072 & 1.462501 & 4.136579 & 11.7000 & 33.09263 & 93.6000 \\
\hline 9 & 0.017424 & 0.156820 & 0.470461 & 1.411382 & 4.234145 & 12.7024 & 38.10731 & 114.321 \\
\hline 10 & 0.013713 & 0.137129 & 0.43364 & 1.371299 & 4.336395 & 13.7129 & 43.36395 & 137.129 \\
\hline 11 & 0.011066 & 0.121721 & 0.403704 & 1.338936 & 4.440749 & 14.7282 & 48.84823 & 162.011 \\
\hline 12 & 0.009113 & 0.109353 & 0.37881 & 1.312235 & 4.545715 & 15.7468 & 54.54858 & 188.961 \\
\hline 13 & 0.007632 & 0.099215 & 0.357724 & 1.289792 & 4.650411 & 16.7672 & 60.45534 & 217.974 \\
\hline 14 & 0.006483 & 0.090760 & 0.339593 & 1.270640 & 4.7543 & 17.7889 & 66.56021 & 249.045 \\
\hline 15 & 0.005574 & 0.083606 & 0.323804 & 1.254088 & 4.857064 & 18.8113 & 72.85595 & 282.169 \\
\hline
\end{tabular}

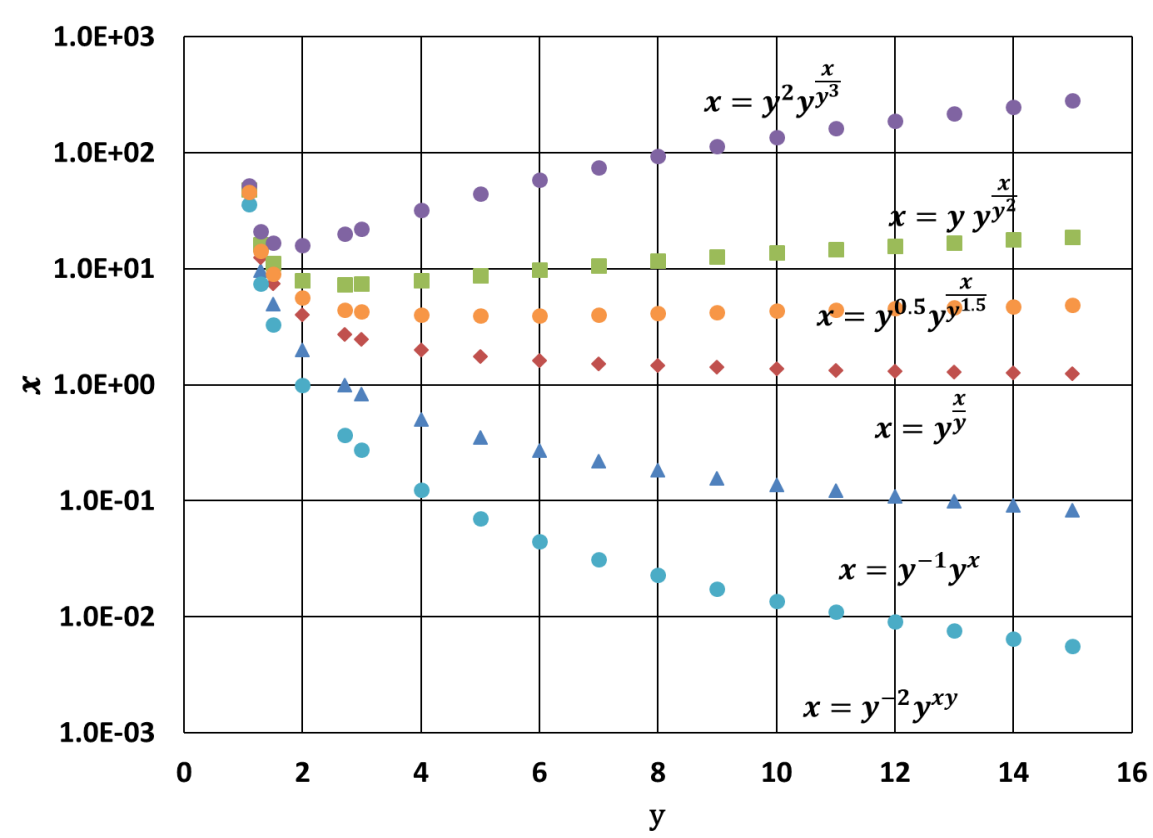

Figure 2. Plots of $x$ vs $y$ for the series of equations, $x=y^{n} y^{\left(\frac{x}{y^{n+1}}\right)}$ for $n=2,1,0,0.5,-1$ and -2 . 
Let

$$
t=-\ln x
$$

Then (1.3) becomes

$$
\begin{gathered}
\frac{-t}{\ln y}=\frac{\mathrm{e}^{-t}}{y^{n+1}}+n \\
(t+n \ln y) \mathrm{e}^{t}=\frac{-\ln y}{y^{n+1}} \\
(t+n \ln y) \mathrm{e}^{t+n \ln y}=\frac{-\mathrm{e}^{n \ln y} \ln y}{y^{n+1}} \\
(t+n \ln y) \mathrm{e}^{t+n \ln y}=\frac{-\ln y}{y} \\
(t+n \ln y)=W\left(\frac{-\ln y}{y}\right)
\end{gathered}
$$

Substituting $-\ln x$ for $t$

$$
(-\ln x+n \ln y)=W\left(\frac{-\ln y}{y}\right)
$$

Using the Equation (1.3)

$$
-\frac{x \ln y}{y^{n+1}}=W\left(\frac{-\ln y}{y}\right)
$$

Hence the solution to Equation (1.3) is

$$
x=\frac{W\left(\frac{-\ln y}{y}\right)}{\left(-\frac{\ln y}{y^{n+1}}\right)}=\frac{y^{n} W\left(\frac{-\ln y}{y}\right)}{\left(-\frac{\ln y}{y}\right)}
$$

If $n=0$, the Equation (1.1) $x=y^{n} y^{\left(\frac{x}{y^{n+1}}\right)}$ becomes Equation (1.7) $x=y^{\left(\frac{x}{y}\right)}$.

Using the solution in the Equation (3.1), the analytical solution in terms of Lambert- $\mathrm{W}$ function is

$$
x=\frac{W\left(-\frac{\ln (y)}{y}\right)}{\left(-\frac{\ln (y)}{y}\right)}
$$

In Equation (3.2), if $y=\mathrm{e}, \quad x=\frac{W\left(-\frac{1}{\mathrm{e}}\right)}{\left(-\frac{1}{\mathrm{e}}\right)}$.

But $W\left(\frac{1}{\mathrm{e}}\right)=-1[6]$.

Hence $x=\mathrm{e}$, the result in Table 1 . 
If $n=0$ and $y=2$ in Equation (3.2), the solutions in Table 1 and Equation (1.7) gives

$$
2^{4}=4^{2} \text { and } \frac{\ln 2}{2}=\frac{\ln 4}{4}=-0.346 .
$$

Equation (3.1) gives

$$
\begin{aligned}
& 4=\frac{W\left(-\frac{\ln (2)}{2}\right)}{\left(-\frac{\ln (2)}{2}\right)}=\frac{W(-0.346)}{-0.346} \\
& 2=\frac{W\left(-\frac{\ln (4)}{4}\right)}{\left(-\frac{\ln (4)}{4}\right)}=\frac{W(-0.346)}{-0.346}
\end{aligned}
$$

$W(-0.346)$ is double valued with -0.693 and -1.386 .

If we substitute the solutions for $n=0$ and $y=10$ from Table 1 to Equation (3.2);

$$
\begin{gathered}
W\left(-\frac{\ln (1.37129)}{1.37129}\right)=10\left(-\frac{\ln (1.37129)}{1.37129}\right) \\
W(-0.2302)=-2.302
\end{gathered}
$$

Since $x$ and $y$ are symmetric in Equation (1.7)

$$
\begin{gathered}
W\left(-\frac{\ln (10)}{10}\right)=1.371289\left(-\frac{\ln (10)}{10}\right) \\
W(-0.2302)=-0.3157
\end{gathered}
$$

The $W(x)$ has two real values for $-1 / \mathrm{e} \leq x<0 \quad[1]$.

If $n=-1$, the Equation (1.1) $x=y^{n} y^{\left(\frac{x}{y^{n+1}}\right)}$ becomes Equation (1.8) $x=y^{-1} y^{x}$ or $x y=y^{x}$.

Using the solution in the Equation (3.1), the analytical solution in terms of the Lambert-W function is

$$
x=\frac{W\left(-\frac{\ln y}{y}\right)}{-\ln y}
$$

If $y=\mathrm{e}, x=\frac{W\left(-\frac{1}{\mathrm{e}}\right)}{-\ln \mathrm{e}}$.

But $W\left(\frac{-1}{e}\right)=-1$, Hence $x=1$, the result in Table 1 .

In Table 1, for any value of $n \quad y=\mathrm{e}, x=\mathrm{e} \times \mathrm{e}^{n}$, the trivial and nontrivial solutions coincide.

$$
y=10, x=1.371289 \times 10^{n}
$$


Using the solution in Equation (3.1), for any $y$ the solution $x$ can be written as

$$
x(n=0) \times y^{n}
$$

Plots of $\ln x$ vs $n$ shown in Figure 3 are linear as expected from Equation (3.1).

The $\ln x$ vs $n$ lines for different $y$ values are crossing near the point $(0.5,1.4)$. This indicates the solutions for $n=0.5$ have little dependency on $y$ for $y \geq e$. This is also evident in the numerical results for $n=0.5$ in Table 1 and in the plot of $x=y^{0.5} y^{\frac{x}{1.5}}$ in Figure 2 .

\section{Solutions $x$ in Base $y$}

The solutions $x$ in Table 1 can be written in base $y,\left(x_{y}\right)$ to indicate the general pattern.

For any valued of $n, x_{y}$ can be written as

$$
x_{y}(n=0) \times 10^{n}
$$

For $n=2$, the solutions $x$ written in base $y, x_{y}$ shown in Table 2 .

For $y>11$, the $x_{y}$ are written using the hex notation.

There is a sharp change in the value of the $x_{y}$ at $y=4$.

For $n=2$, plot $x_{y}$ vs $y$, for $5 \leq y \leq 11$ is shown in Figure 4 .

\section{Connection to the Fine Structure Constant}

In Equation (1.1), when $n=2$ and $y=10$, the equation becomes $x=10^{2} \times 10^{\frac{x}{10^{3}}}$ and the solution is

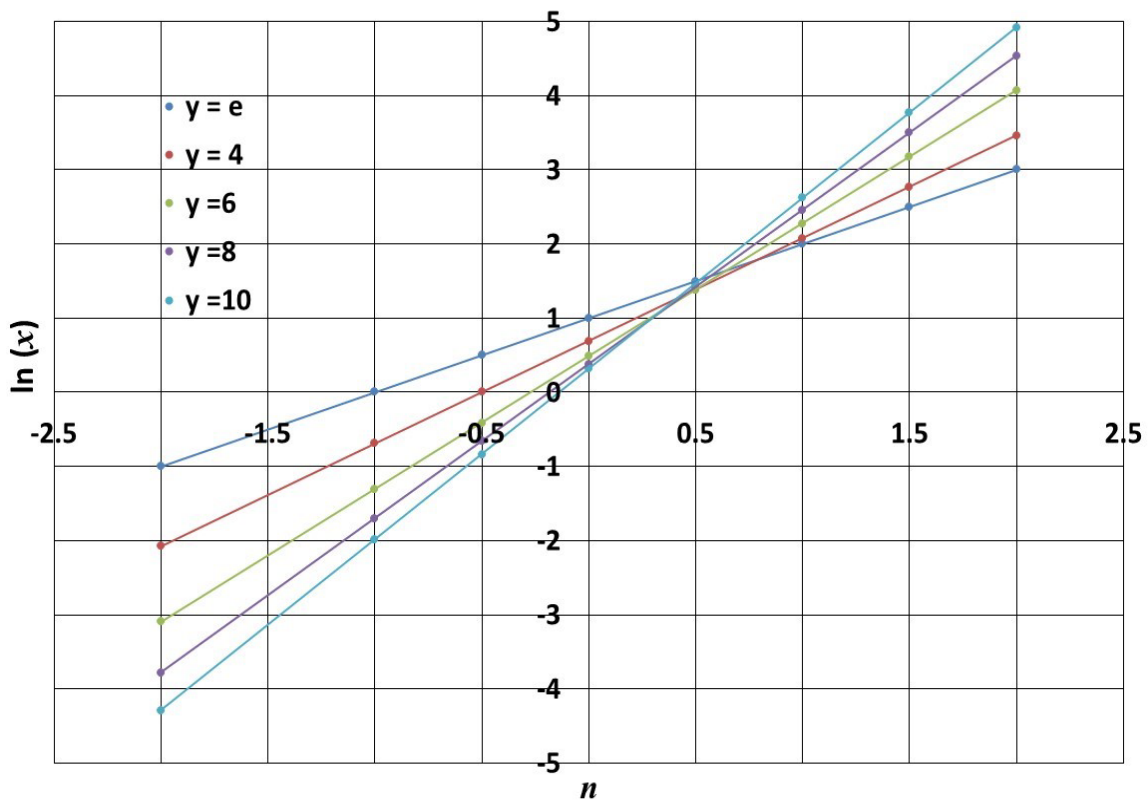

Figure 3. Plots of $\ln x$ vs $n$. 
Table 2. Solutions $x$ in base $y\left(x_{y}\right)$.

\begin{tabular}{|c|c|}
\hline$y$ & $\begin{array}{c}x \text { written in base } y \text { for } n=2 \\
x_{y}\end{array}$ \\
\hline 2 & 10000.000 \\
\hline $\mathrm{e}$ & 1000.000 \\
\hline 3 & 211.022 \\
\hline 4 & 200.000 \\
\hline 5 & 134.030 \\
\hline 6 & 134.250 \\
\hline 7 & 134.656 \\
\hline 8 & 135.463 \\
\hline 9 & 136.281 \\
\hline 10 & 137.129 \\
\hline 11 & 138.014 \\
\hline 12 & 138.B60 \\
\hline 13 & 139.C67 \\
\hline 14 & 13B.090 \\
\hline 15 & 13C. 280 \\
\hline 16 & 13D.590 \\
\hline 17 & 13E.9A0 \\
\hline 18 & 13F.F00 \\
\hline
\end{tabular}

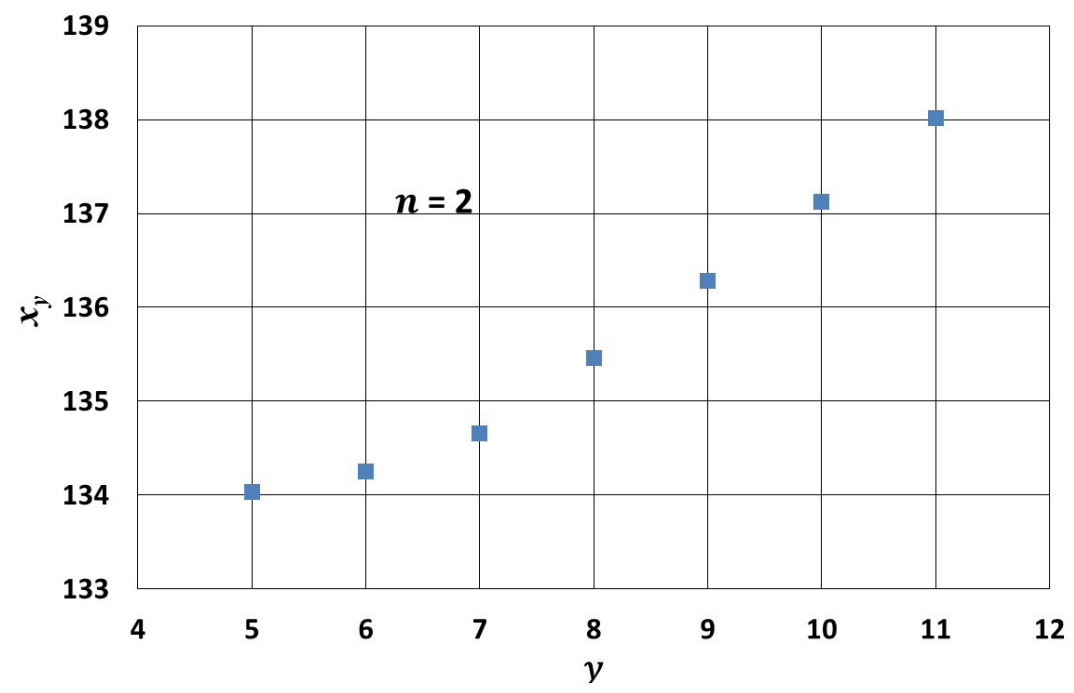

Figure 4. Plot of $x_{y}$ vs $y$ for $n=2$.

$$
x=\frac{10^{2} W\left(\frac{-\ln 10}{10}\right)}{\left(-\frac{\ln 10}{10}\right)}=137.129
$$


The solution 137.129 is close to the inverse of the fine structure constant 137.036 [14]-[21] which is dimensionless.

The inverse of the fine structure constant $\alpha^{-1}$ is given by the expression

$$
\alpha^{-1}=\frac{4 \pi \varepsilon_{o} \hbar c}{e^{2}}=137.036
$$

where;

$$
\begin{aligned}
& \hbar=1.0545718 \times 10^{-34} \mathrm{~J} \cdot \mathrm{s}, \text { reduced Planck constant; } \\
& c=2.99792458 \times 10^{8} \mathrm{~m} \cdot \mathrm{s}^{-1}, \text { speed of light in vacuum; } \\
& \varepsilon_{0}=8.854187817 \times 10^{-12} \mathrm{~F} \cdot \mathrm{m}^{-1} \text {, electric constant; } \\
& e=1.6021766208 \times 10^{-19} \mathrm{C} \text {, elementary charge; } \\
& \alpha^{-1} \text {, dimensionless constant [22]. }
\end{aligned}
$$

In a recent publication Eaves [23] suggested an equation relating $G$ and $\alpha$;

$$
\frac{\alpha q^{2}}{8 \pi^{2} G m_{e}^{2}} \approx \exp \left(\frac{2}{3 \alpha}\right)
$$

where;

$$
\begin{gathered}
G=6.67408 \times 10^{-11} \mathrm{~m}^{3} \cdot \mathrm{kg}^{-1} \cdot \mathrm{s}^{-2}, \text { gravitational constant; } \\
m_{e}=9.10938356 \times 10^{-31} \mathrm{~kg}, \text { electron mass. } \\
q^{2}=\frac{e^{2}}{4 \pi \varepsilon_{0}}
\end{gathered}
$$

By substituting the expression for $\alpha$ in Equation (6.3) we get

$$
\frac{e^{4}}{32 \pi^{3} \varepsilon_{0}^{2} G m_{e}^{2} \hbar c} \approx \exp \left(\frac{2}{3 \alpha}\right)
$$

Using Equation (6.2), the Equation (6.4) becomes

$$
\alpha^{-1} \approx \frac{64 \pi^{4} \varepsilon_{0}^{3} \hbar^{2} c^{2} G m_{e}^{2}}{e^{6}} \exp \left(\frac{\alpha^{-1}}{1.5}\right)
$$

Substituting numerical values for the pre-exponent,

$$
\begin{gathered}
\alpha^{-1} \approx 1.59947 \times 10^{-27} \exp \left(\frac{\alpha^{-1}}{1.5}\right) \\
\alpha^{-1} \approx 1.59947 \times 10^{-27} \times 10^{\left(\frac{\alpha^{-1}}{3.4538}\right)}
\end{gathered}
$$

By taking the power of (1/289.5) on both sides of the Equation (6.8) and writing the equation for $\alpha^{-1}$ yields

$$
\alpha^{-1} \approx 106.6 \times 10^{\left(\frac{\alpha^{-1}}{1000}\right)}
$$

The Equation (6.8) is approximately the same as the equation $x=10^{2} \times 10^{\frac{x}{10^{3}}}$. The only difference is the $10^{2}$ is 106.6 in Equation (6.8). But the Equation (6.8) based on the Equation (6.3) is only an approximate equation.

The value $1.59947 \times 10^{-27}$ in Equation (6.6) is approximately equal to the $\alpha_{G}^{1 / 1.5}, \quad \alpha_{G}$ defined by Jentschura [20]. 


$$
\alpha_{G}=\frac{G m_{e} m_{p}}{\hbar c}=3.21 \times 10^{-42}
$$

Hence the Equation (6.7) can be written as

$$
\alpha^{-1} \approx \alpha_{G}^{1 / 1.5} \exp \left(\frac{\alpha^{-1}}{1.5}\right)
$$

\section{Conclusions}

An equation in the form of $x=y^{n} y^{\left(\frac{x}{y^{n+1}}\right)}$ was solved graphically, numerically and analytically.

The plots of numerical solution $x$ vs $y$ indicate sharp turning points for $y$ values in-between 1 to 2 .

The analytical solution was found in terms of Lambert-W function as

$$
x=\frac{y^{n} W\left(\frac{-\ln y}{y}\right)}{\left(-\frac{\ln y}{y}\right)}
$$

The numerical solutions can be written as $x(n=0) \times y^{n}$.

The numerical solutions can also be written in base $y$ as $x_{y}(n=0) \times 10^{n}$. For $y \geq 5 x_{y}(n=0)$ is a universal number approximately equal to 1.37 .

$$
\text { If } n=2 \text { and } y=10 \text {, the solution } x=\frac{10^{2} W\left(\frac{-\ln 10}{10}\right)}{\left(-\frac{\ln 10}{10}\right)}=137.129 \text { (rounded) is }
$$

close to the inverse of the fine structure constant value, 137.036.

The equation $x=10^{2} \times 10^{\frac{x}{10^{3}}}$ which gives the solution close to the fine structure constant can be derived from the equation $\frac{\alpha q^{2}}{8 \pi^{2} G m_{e}^{2}} \approx \exp \left(\frac{2}{3 \alpha}\right)$ suggested by Eaves.

The derivation resulted in an equation $\alpha^{-1} \approx \alpha_{G}^{1 / 1.5} \exp \left(\frac{\alpha^{-1}}{1.5}\right)$.

\section{References}

[1] Corless, R.M., et al. (1996) On the LambertW Function. Advances in Computational Mathematics, 5, 329-359. https://doi.org/10.1007/BF02124750

[2] Dence, T.P. (2013) A Brief Look into the Lambert W Function. Applied Mathematics, 4, 887. https://doi.org/10.4236/am.2013.46122

[3] Kalman, D. (2001) A Generalized Logarithm for Exponential-Linear Equations. The College Mathematics Journal, 32, 2-14. https://doi.org/10.1080/07468342.2001.11921844

[4] Fukushima, T. (2013) Precise and Fast Computation of Lambert W-Functions without Transcendental Function Evaluations. Journal of Computational and Ap- 
plied Mathematics, 244, 77-89. https://doi.org/10.1016/j.cam.2012.11.021

[5] Valluri, S.R., et al. (2009) The Lambert W Function and Quantum Statistics. Journal of Mathematical Physics, 50, Article Id: 102103. https://doi.org/10.1063/1.3230482

[6] Valluri, S.R., Jeffrey, D.J. and Corless, R.M. (2000) Some Applications of the Lambert W Function to Physics. Canadian Journal of Physics, 78, 823-831.

[7] Scott, T.C., Mann, R. and Martinez Ii, R.E. (2006) General Relativity and Quantum Mechanics: Towards a Generalization of the Lambert W Function A Generalization of the Lambert W Function. Applicable Algebra in Engineering, Communication and Computing, 17, 41-47. https://doi.org/10.1007/s00200-006-0196-1

[8] Jain, A. and Kapoor, A. (2004) Exact Analytical Solutions of the Parameters of Real Solar Cells Using Lambert W-Function. Solar Energy Materials and Solar Cells, 81, 269-277. https://doi.org/10.1016/j.solmat.2003.11.018

[9] Goličnik, M. (2012) On the Lambert W Function and Its Utility in Biochemical Kinetics. Biochemical Engineering Journal, 63, 116-123. https://doi.org/10.1016/j.bej.2012.01.010

[10] Kitis, G. and Vlachos, N. (2013) General Semi-Analytical Expressions for TL, OSL and Other Luminescence Stimulation Modes Derived from the OTOR Model Using the Lambert W-Function. Radiation Measurements, 48, 47-54. https://doi.org/10.1016/j.radmeas.2012.09.006

[11] Barry, D., et al. (1993) A Class of Exact Solutions for Richards' Equation. Journal of Hydrology, 142, 29-46. https://doi.org/10.1016/0022-1694(93)90003-R

[12] Weisstein, E.W. (2002) Lambert W-Function.

[13] Gnanarajan, S. (2017) Solutions of the Exponential Equation $y^{x / y}=x$ or $\ln x / x=\ln y / y$ and Fine Structure Constant. Journal of Applied Mathematics and Physics, 5, 386. https://doi.org/10.4236/jamp.2017.52034

[14] Kinoshita, T. (1996) The Fine Structure Constant. Reports on Progress in Physics, 59, 1459. https://doi.org/10.1088/0034-4885/59/11/003

[15] Dirac, P.A. (1937) The Cosmological Constants. Nature, 139, 323. https://doi.org/10.1038/139323a0

[16] Bouchendira, R., et al. (2011) New Determination of the Fine Structure Constant and Test of the Quantum Electrodynamics. Physical Review Letters, 106, Article ID: 080801. https://doi.org/10.1103/PhysRevLett.106.080801

[17] Hammer, E. (2006) Physical and Mathematical Meaning of the Alpha Constant, Einstein's Equation, and Planck Dimensions. Industry Applications Conference, Tampa, 8-12 October 2006. https://doi.org/10.1109/IAS.2006.256848

[18] Kragh, H. (2003) Magic Number: A Partial History of the Fine-Structure Constant. Archive for History of Exact Sciences, 57, 395-431.

[19] Sandvik, H.B., Barrow, J.D. and Magueijo, J. (2002) A Simple Cosmology with a Varying Fine Structure Constant. Physical Review Letters, 88, Article ID: 031302. https://doi.org/10.1103/PhysRevLett.88.031302

[20] Jentschura, U.D. (2014) Fine-Structure Constant for Gravitational and Scalar Interactions. Physical Review A, 90, Article ID: 022112.

https://doi.org/10.1103/PhysRevA.90.022112

[21] Jentschura, U.D. and Nándori, I. (2014) Attempts at a Determination of the Fine-Structure Constant from First Principles: A Brief Historical Overview. The European Physical Journal H, 39, 591-613. https://doi.org/10.1140/epjh/e2014-50044-7

[22] Mohr, P.J., Newell, D.B. and Taylor, B.N. (2016) CODATA Recommended Values 
of the Fundamental Physical Constants: 2014. Journal of Physical and Chemical Reference Data, 45, Article ID: 043102. https://doi.org/10.1063/1.4954402

[23] Eaves, L. (2018) A Model to Inter-Relate the Values of the Quantum Electrodynamic, Gravitational and Cosmological Constants. 